\title{
Uncovering Intrinsic Connectional Architecture of Functional Networks in Awake Rat Brain
}

\author{
Zhifeng Liang, Jean King, and Nanyin Zhang \\ Center for Comparative Neuroimaging, Department of Psychiatry, University of Massachusetts Medical School, Worcester, Massachusetts 01655
}

Intrinsic connectional architecture of the brain is a crucial element in understanding the governing principle of brain organization. To date, enormous effort has been focused on addressing this issue in humans by combining resting-state functional magnetic resonance imaging (rsfMRI) with other techniques. However, this research area is significantly underexplored in animals, perhaps because of confounding effects of anesthetic agents used in most animal experiments on functional connectivity. To bridge this gap, we have systematically investigated the intrinsic connectional architecture in the rodent brain by using a previously established awake-animal imaging model. First, group independent component analysis was applied to the rsfMRI data to extract elementary functional clusters of the brain. The connectional relationships between these clusters, as evaluated by partial correlation analysis, were then used to construct a graph of whole-brain neural network. This network exhibited the typical features of small-worldness and strong community structures seen in the human brain. Finally, the whole-brain network was segregated into community structures using a graph-based analysis. The results of this work provided a functional atlas of intrinsic connectional architecture of the rat brain at both intraregion and interregion levels. More importantly, the current work revealed that functional networks in rats are organized in a nontrivial manner and conserve fundamental topological properties that are also seen in the human brain. Given the high psychopathological relevance of network organization of the brain, this study demonstrated the feasibility of studying mechanisms and therapies of multiple neurological and psychiatric diseases through translational research.

\section{Introduction}

The effort to understand the connectional architecture of the brain has benefited tremendously from the advent of restingstate functional magnetic resonance imaging (rsfMRI). rsfMRI is a technique that noninvasively measures functional connectivity without external stimulation based on spontaneous lowfrequency fluctuations of the fMRI signal (Biswal et al., 1995; Fox and Raichle, 2007). Using this technique, resting-state functional connectivity (RSFC) has been consistently revealed in multiple networks of the human brain (Biswal et al., 1995; Greicius et al., 2003; Fox et al., 2005), and has been shown to be altered by the effects of sleep, anesthesia, and aging (Stevens et al., 2008; Horovitz et al., 2009). Recent studies have also delineated significant influences of various pathological conditions on RSFC (Greicius et al., 2007), indicating vital neurobiological and psychopathological relevance (Kennedy et al., 2006; Albert et al., 2009).

Well documented properties of intraregional and interregional connectivity make it extremely intriguing to extend the RSFC research at local brain regions to global brain networks.

\footnotetext{
Received Aug. 31, 2010; revised Jan. 11, 2011; accepted Jan. 14, 2011.

This work was supported by the National Institutes of Health (NIH) Grants 1R01 MH067096-02 and 5R01DA021846-02, and the institutional fund from the University of Massachusetts Medical School. The contents are solely the responsibility of the authors and do not necessarily represent the official views of the NIH. We thank Dr. Wei Huang for her technical assistance. We also thank the reviewers for their insightful comments.

Correspondence should be addressed to Dr. Nanyin Zhang, Center for Comparative Neuroimaging, Department of Psychiatry, University of Massachusetts Medical School, 55 Lake Avenue North, Worcester, MA 01655. E-mail: Nanyin.Zhang@umassmed.edu.

DOI:10.1523/JNEUROSCI.4557-10.2011

Copyright $\odot 2011$ the authors $\quad 0270-6474 / 11 / 313776-08 \$ 15.00 / 0$
}

Using graph-based analysis, separately identified brain networks subserving different functions in humans were found to topologically organize in a nontrivial manner to support efficient information processing (Wang et al., 2010). Graph theoretical approaches in rsfMRI use anatomically or functionally defined regions of interest (ROIs) as vertices, and connectivity between ROIs as edges. These approaches have revealed that the human brain's networks are characterized by properties of small-world topology, highly connected hub, and high modularity (Bullmore and Sporns, 2009). These findings are crucial because they identified the governing principle of the network organization of the human brain; the same methods can be used to examine alterations of topological configuration of the brain in response to external stimulation or in different pathological conditions (Liu et al., 2008; Bassett and Bullmore, 2009). Therefore, these methods may serve as a potential biomarker of various mental disorders.

To date, the majority of studies on intrinsic connectional organization of the brain have been conducted in humans. Systematic investigations of this issue in different animal models have been significantly underexplored (Vincent et al., 2007; Pawela et al., 2008; Schwarz et al., 2009), partially attributed to confounding effects of anesthetic agent used in animal studies on RSFC (Massimini et al., 2005; Lu et al., 2007; Liu et al., 2010). Consequently, it is very important to explore RSFC in awake animals not only because it can provide invaluable information regarding intrinsic connectional architecture of the animal brain and its reconfiguration in response to cognitive and emotional stimuli, but also may provide a unique window to explore comparative 
functional anatomy between species. Moreover, understanding connectional architecture in animals will allow us to investigate multiple psychiatric and neurological diseases using translational models. Recently, we have successfully demonstrated the feasibility of mapping RSFC in awake rats (Zhang et al., 2010) based on an awake-animal imaging model that has been well established in our laboratory (King et al., 2005; Ferris et al., 2006). Using the same animal model here, we have characterized the intrinsic network architecture in the awake rat.

\section{Materials and Methods}

Animals. Sixteen adult male Long-Evans rats $(350-450 \mathrm{~g})$ were obtained from Charles River Laboratories. Animals were housed in Plexiglas cages (two per cage) and maintained in ambient temperature $\left(22-24^{\circ} \mathrm{C}\right)$ on a $12 \mathrm{~h}$ light:12 h dark schedule. Food and water were provided ad libitum. All studies were approved by Institutional Animal Care and Use Committee of the University of Massachusetts Medical School.

Acclimation procedure. All rats were acclimated to MRI restraint and noise as previously described (King et al., 2005; Ferris et al., 2006). Briefly, rats were anesthetized with isoflurane and secured in Plexiglas stereotaxic head holder using plastic ear-bars. Emla cream (AstraZeneca) was applied tropically to minimize pain of mechanical restraint. Animals were then placed into black opaque tube mock scanner with taperecorded scanner noises. Animals were acclimated for $8 \mathrm{~d}$, one session per day. The time for exposure was increased from $15 \mathrm{~min}$ on the first day to 90 min on days 6,7 , and 8 , with an increment of 15 min per day (King et al., 2005).

Animal preparation. Under short-acting isoflurane gas, the animal was fitted into a head restrainer with a built-in coil. The head was placed into the cylindrical head-holder with the canines secured over a bite bar, the nose secured with a nose clamp, and ears positioned inside the headholder with adjustable screws fitted into lateral sleeves. The body of the animal was placed into a body restrainer that allowed unrestricted respiration. After the animal was set up, the isoflurane gas was removed and the restraining system was positioned in the magnet. Animals were fully conscious within $10-15 \mathrm{~min}$.

MR experiments. All experiments were performed on a Bruker 4.7T/40 $\mathrm{cm}$ horizontal magnet interfaced with a Biospec Bruker console. A dual ${ }^{1} \mathrm{H}$ radiofrequency coil configuration (Insight NeuroImaging Systems), consisting of a volume coil for exciting MRI signal and a surface coil for receiving MRI signal, was used. The volume and surface coils were actively tuned and detuned to prevent mutual coil coupling.

For each session, anatomical images were acquired with a fast spinecho sequence [rapid acquisition with relaxation enhancement (RARE)] with the following parameters: repetition time (TR), $2125 \mathrm{~ms}$; RARE factor, 8; echo time (TE), $50 \mathrm{~ms}$; matrix size, $256 \times 256$; field of view (FOV), $3.2 \times 3.2 \mathrm{~cm}^{2}$; slice number, 18 ; slice thickness, $1 \mathrm{~mm} . \mathrm{T}_{2}{ }^{*}$ weighted gradient-echo images coving the whole brain were then acquired using the echo-planar imaging (EPI) sequence with following parameters: TR, $1 \mathrm{~s}$; TE, $30 \mathrm{~ms}$; flip angle, $60^{\circ}$; matrix size, $64 \times 64$; FOV, $3.2 \times 3.2 \mathrm{~cm}^{2}$; slice number, 18 ; slice thickness, $1 \mathrm{~mm}$. Two hundred EPI volumes were acquired for each run, and six runs were obtained for each session. Rats were in resting state during all imaging sessions.

Preprocessing of imaging data. Imaging data were preprocessed using Medical Image Visualization and Analysis (MIVA, http://ccni.wpi.edu/), Statistical Parametric Mapping (SPM8) software (Wellcome Department of Cognitive Neurology, London, UK), and MATLAB (MathWorks). All images were first aligned and coregistered with MIVA as previously described (Zhang et al., 2010). After registration, all functional images were preprocessed with steps of motion correction, spatial smoothing (full-width at half-maximum, $1 \mathrm{~mm}$ ), voxelwise linear detrending, and $0.002-0.1 \mathrm{~Hz}$ bandpass filtering. Datasets with excessive motion ( $>0.25 \mathrm{~mm}$, eight runs in total) were discarded, resulting in a total of 88 runs for subsequent analysis.

Independent component analysis. Group independent component analysis (ICA) (Calhoun et al., 2001) was performed using GIFT toolbox (http://www.nitrc.org/projects/gift/). The number of components was set at 40 (Hutchison et al., 2010). The infomax algorithm was used to perform spatial ICA and independent components were scaled to $z$ scores. Time courses of individual components for individual scans were extracted. Among the spatial maps of all 40 components, two were located at CSF areas and were identified as artifactual components.

Direct connectivity and graph theory analysis. Time courses of $40 \mathrm{com}-$ ponents were used in direct connectivity analysis. For each individual RSFC run, the partial correlation coefficient between time courses of each pair of components was calculated, conditioning on time courses of the other 38 components. This step yielded a $40 \times 40$ partial correlation matrix for each run. Partial correlation coefficients ( $r$ values) were transformed to $z$ scores and then averaged across all runs and across all animals. The final partial correlation matrix was generated by transforming the averaged $z$ scores back to the $r$ values. Each element of this matrix represented the strength of direct connectivity between two components. We only focused on positive partial correlation coefficients, although negative coefficients were also detected. The significance of direct connectivity was calculated by using a one-sample $t$ test and thresholded at $p<0.01$ ( $n=88$, uncorrected $)$, based on all 88 partial correlation matrices. The two artifactual components did not show significant connections with other components, and thus were eliminated in further graph-theory analyses. As a result, a $38 \times 38$ adjacency matrix was generated with each element, $a_{i j}$, describing the significant direct connection between each two components based on the $p$ value:

$$
a_{i j}=\left\{\begin{array}{ll}
1, & \text { if component } i \text { and } j \text { are conncted (i.e., } p<0.01) \\
0, & \text { otherwise }
\end{array} .\right.
$$

Based on this adjacency matrix, the community structure of the rat brain was obtained by using the spectral partitioning method (Newman, 2006). Modularity $\mathrm{Q}$ is defined as follows:

$$
Q=\frac{1}{4 m} \sum_{i j}\left(a_{i j}-\frac{k_{i} k_{j}}{2 m}\right) \delta\left(c_{i}, c_{j}\right),
$$

where $m$ is the total number of edges in the network, $k_{i}$ and $k_{j}$ are the degree of each vertex, $c_{i}$ is the group to which vertex $i$ belongs, and $\delta\left(c_{i}, c_{j}\right)$ is the Kronecker delta symbol.

The partitioning analysis followed the procedure used previously (Newman, 2006) and consisted of two steps. In the first step, we obtained a single solution of partitioning by using the spectral approach based on the leading eigenvector of the modularity matrix (Newman, 2006). This step, as pointed out by Newman (2006), gives an excellent guide to the general form that the communities should take. In the second step, we combined the spectral method and the fine-tuning method described by Newman (2006) to further optimize modularity. Considering that the modularity function $Q$, generated by combining the spectral method and the fine-tuning method, is degenerate (Good et al., 2010), we computed a distribution of $Q$ values and a distribution of partitions by permuting the order of nodes in the adjacency matrix before feeding it into the optimization algorithm in the second step. Only the solution consistent over this distribution was reported. The degeneracy of $Q$ is $\sim 2^{k}$, where $k$ is the number of modules (Good et al., 2010). Since three modules were found in the first step, $>2^{k}$ (20) repetitions were made to form the distribution of $Q$ values and partitions. All analyses in the second step were performed using Brain Connectivity Toolbox (BCT) (Rubinov and Sporns, 2010). After partitioning, components belonging to the same module were displayed in the same colors in the figures.

Clustering coefficient and shortest path length. The averaged local clustering coefficient was calculated as

$$
C=\frac{1}{m} \sum_{j=1}^{m} \frac{2 E_{j}}{V_{j}\left(V_{j}-1\right)}
$$

where $E_{j}$ is the number of edges connecting neighbors of vertex $j$ and $V_{j}$ is the number of neighbors of vertex $j$. Pure random networks with same numbers of nodes and edges were constructed based on the Erdős-Rényi model with 100 repetitions. Random networks with the same distribution of degrees as the current rat-brain network were constructed using 


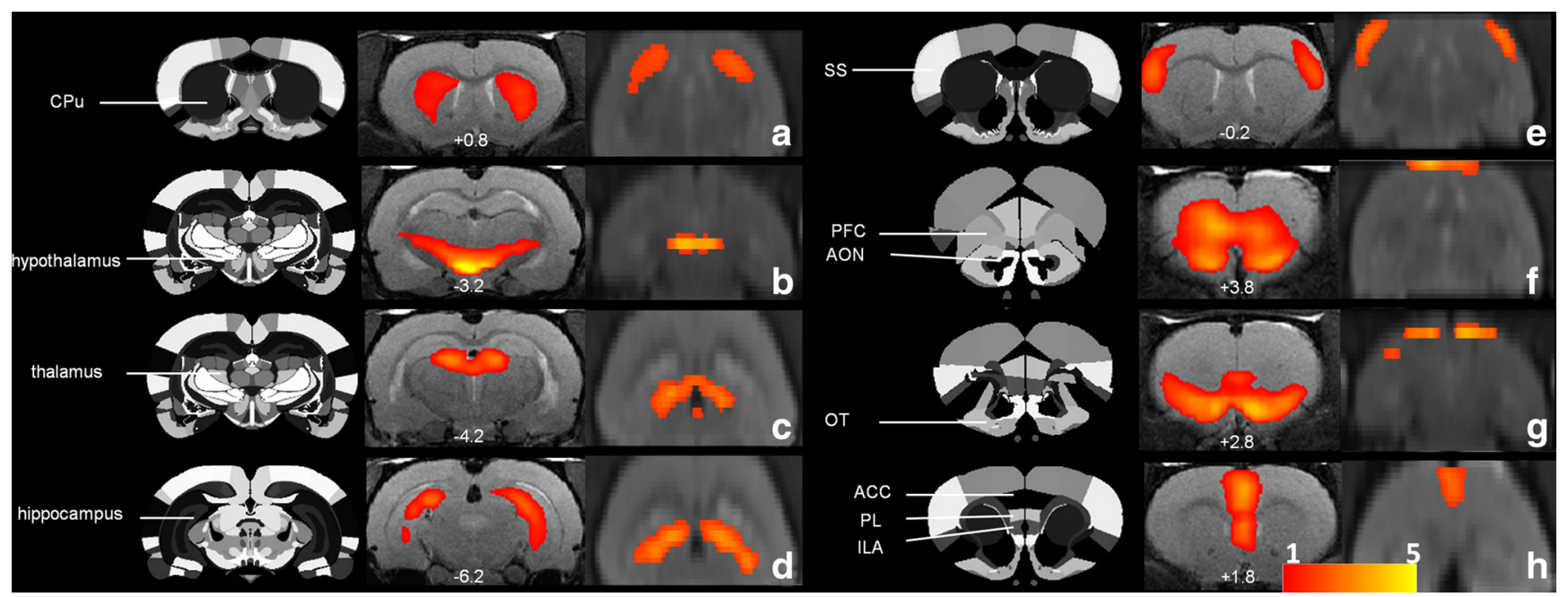

Figure 1. Spatial maps of individual components identified by ICA. $\boldsymbol{a}-\boldsymbol{h}$, Examples of ICA components. Left columns are atlas images. Anatomic regions corresponding to individual ICA components are annotated. Middle columns are individual ICA components overlaid on anatomical images in the coronal view. Distances to bregma (in millimeters) are labeled at the bottom of each image. Right columns are individual ICA components overlaid on anatomical images in the transversal view. SS, somatosensory cortex; PL, prelimbic cortex; ILA, infralimbic cortex.

BCT with 100 repetitions. The averaged minimum path length was calculated as

$$
L=\frac{2}{m \times(m-1)} \sum_{j=1}^{m-1} \sum_{k=j+1}^{m} \min \_ \text {path }(j, k),
$$

where min_path is the shortest path length between vertices $j$ and $k$.

Reproducibility of intercomponent direct connectivity. To estimate the reliability of intercomponent connectivity across animals, we randomly divided data from all animals into two subgroups. The strength of intercomponent connectivity (defined as the amplitude of partial correlation coefficient between two components) between the two subgroups was quantitatively compared using the correlation of intercomponent connectional strength between the two subgroups. This procedure was repeated 100 times and the correlation value averaged across 100 repetitions was reported.

\section{Results}

\section{Elementary clusters of RSFC revealed by group ICA}

Group ICA results were obtained from 16 conscious rats. Most components identified were located in specific anatomical regions as displayed in Figure 1. Figure $1 a$ shows a component located at anatomically well defined bilateral caudate-putamen $(\mathrm{CPu})$. Figure $1, b-e$, represents functional structures of bilateral hypothalamus, thalamus, hippocampus, and somatosensory cortex, respectively. In addition, functionally related regions also tended to cluster into single components. Figure $1 f$ shows a component that includes bilateral prefrontal cortex (PFC) and anterior olfactory nucleus (AON), showing well known reciprocal functional connections of the olfactory bulbs and other olfactoryrelated areas with the prefrontal cortex in conscious rats (Cinelli et al., 1987). Another olfactory-related component was located at olfactory tubercle (OT) (Fig. $1 g$ ). Figure $1 \mathrm{~h}$ shows a complex component composed of anterior cingulate cortex (ACC), prelimbic, and infralimbic cortices, together considered as extended areas of $\mathrm{PFC}$ in the rat.

Figure 2 shows 38 ICA components (excluding two artifactual components) overlaid on anatomical images, revealing the global clustering pattern of RSFC in the rat brain. Bilateral components were dominant of all ICA components identified (24 of 38). In cortical regions, bilateral components (13 in total) were also dominant. The numbers of left and right cortical components were approximately equal (five for left lateral components and six for right components).

\section{Direct connectivity between RSFC clusters calculated by partial correlation}

To evaluate intercomponent connectional relationships, we calculated the direct connectivity between individual components by using partial correlation analysis. The partial correlation coefficient matrix of 40 components averaged across all animals is displayed in Figure 3a. Statistical comparison at the group level revealed the pattern of direct connections between different RSFC clusters (one-sample $t$ test, $p<0.01$ ). To estimate the reliability of intercomponent connectivity across animals, we randomly divided data from all animals into two subgroups. There was a high correlation of intercomponent connectional strength between the two subgroups $\left(r=0.71, p<10^{-6}\right)$ (Fig. $3 b$ ), suggesting great reproducibility in direct connectivity between RSFC clusters. This result did not change when we repeated the same process 100 times (averaged correlation coefficient of 100 repetitions, $\left.r_{\text {avg }}=0.68\right)$.

\section{Graph theory-based analysis of the rat brain networks}

The graph demonstration of significant direct connections between ICA components is shown in Figure $4 a$. The total edge number was 78 , yielding the connection density of $5.55 \%$. The spectral partitioning algorithm, based on the leading eigenvector (Newman, 2006), was applied to this graph (the first step of partitioning; see Materials and Methods) and revealed that the rat whole-brain network was segregated into three modules to achieve maximum modularity $(Q=0.414)$ (Fig. 4). This modularity value was significantly higher than both random networks with same nodes and edges and random networks with same degree distribution $(p<0.01$ for both types of random networks), suggesting a prominent modular structure of intrinsic connectional architecture of the rat brain. Of the three modules, module 1 was dominated by cortical regions, including the dorsal olfactory bulb, motor cortex, somatosensory cortex, insular cortex, and visual cortex (Fig. 4b), indicating strong, direct communications across the cortical ribbon in the rat (Zhang et al., 2010). Module 2 included the olfactory system, PFC, ACC, CPu, poste- 

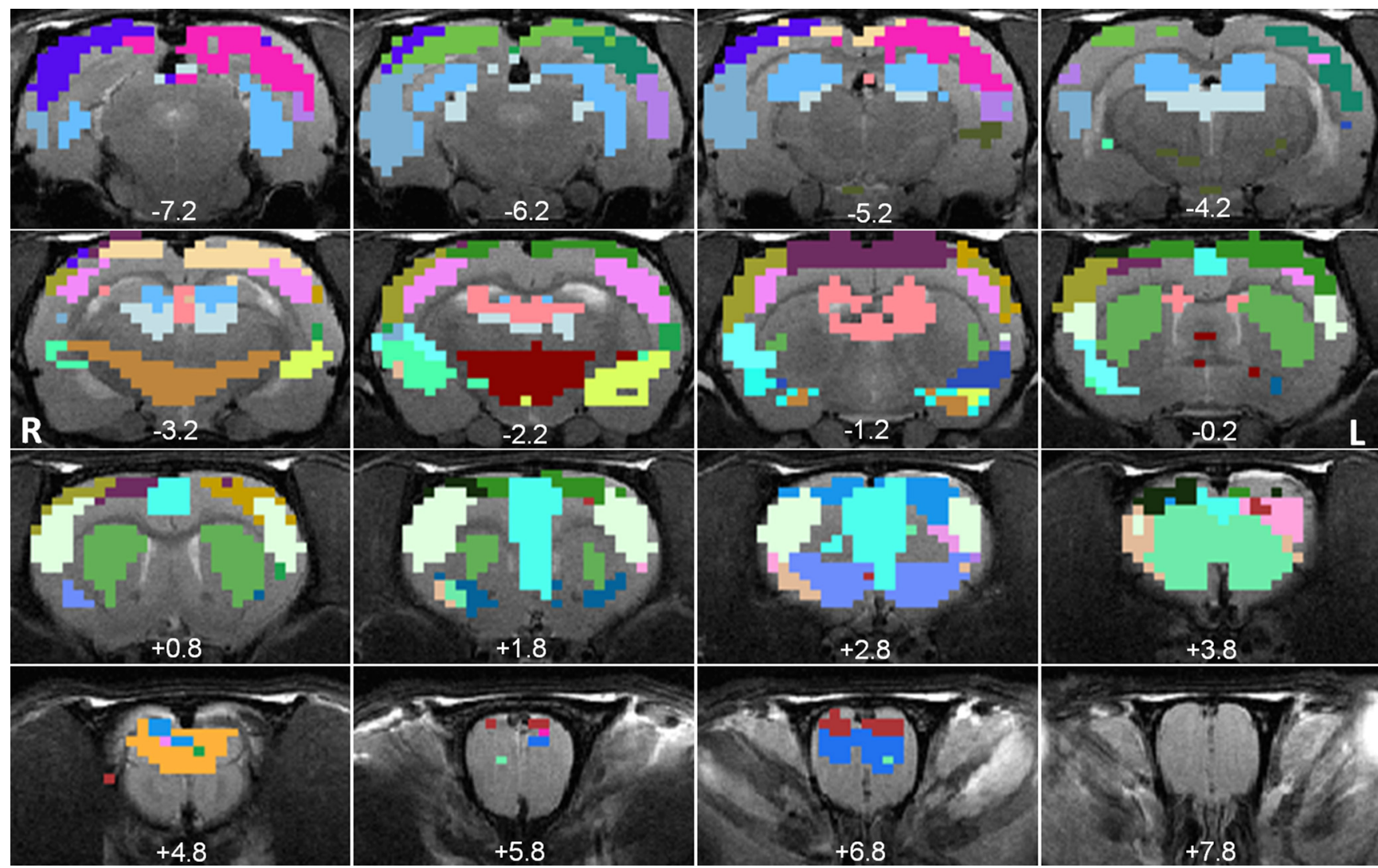

Figure 2. The spatial pattern of 38 group ICA components (excluding two artifactual components). Individual components are displayed with distinct colors. Distance to bregma (in millimeters) for each imaging slice is labeled at the bottom of each image.
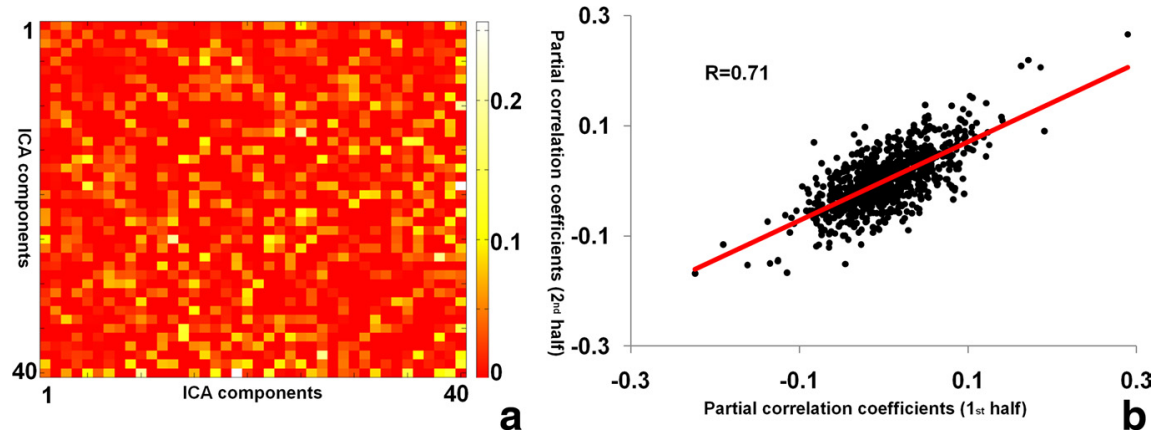

Figure 3. Intercomponent connectional relationships. $\boldsymbol{a}$, The partial correlation coefficient matrix averaged across all rats. Partial correlation coefficients ( $r$ values) were first transformed to $z$ scores and then averaged across all runs and across all animals. The final partial correlation matrix was generated by transforming the averaged $z$ scores back to the $r$ values. All diagonal values were set to zero. $\boldsymbol{b}$, Correlation of direct connectional strength ( $r$ values) between two randomly divided subgroups. The high correlation coefficient (0.71) suggests that intercomponent connections across animals are highly reproducible.

rior somatosensory cortex, thalamus, hypothalamus, hippocampus, and auditory cortex. This module highlighted the integration of sensory input, cognitive processing, and output (Paxinos, 2004). Module 3 consisted of the PFC, insular cortex, amygdala, hypothalamus, and auditory cortex. This module might be related to emotion and autonomic regulation in the conscious rat (Paxinos, 2004).

To further maximize the final value of modularity, finetuning stages, as described by Newman's (2006) spectral partitioning analysis, were included in the second step of the partitioning procedure. Considering that the modularity function $Q$ is degenerate and leads to multiple solutions of graph partitioning (Good et al., 2010), we computed the distribution of $Q$ values and partitions. The distribution of $Q$ values ranged from
0.392 to 0.429 with a mean value of 0.416 , which only slightly improved the $Q$ value of 0.414 obtained in the first step. In all repetitions, the majority yielded four modules (12 of 20 repetitions). The major pattern of partitioning showed very high stability. Consistent with the partitioning result from the first step, two modules, identical to the green and yellow modules (Fig. 4), were highly consistent in all 20 partitions with minimal variation. The yellow module was found in all repetitions and the green module was found in 19 of 20 repetitions. However, the red module was less stable and tended to be further divided into two submodules (Fig. 5). The first submodule was found in 14 of 20 repetitions and the second submodule was found in 13 or 20 repetitions. This reduced stability of the cortical module might indicate higher complexity of cortical network organization.

Furthermore, the connectional architecture of the rat brain showed typical features of small-worldness, characterized by high clustering coefficient and short minimum path length. When compared to pure random networks with the same numbers of nodes and edges, the ratio of clustering coefficient $\left(C / C_{\text {random }}\right)$ is 1.7 and the ratio of minimum path length $\left(L / L_{\text {random }}\right)$ is 1.08 , indicating a higher level of clustering than and a similar minimum path length to pure randomized networks. The ratios of these two metrics, compared with a random network with the 

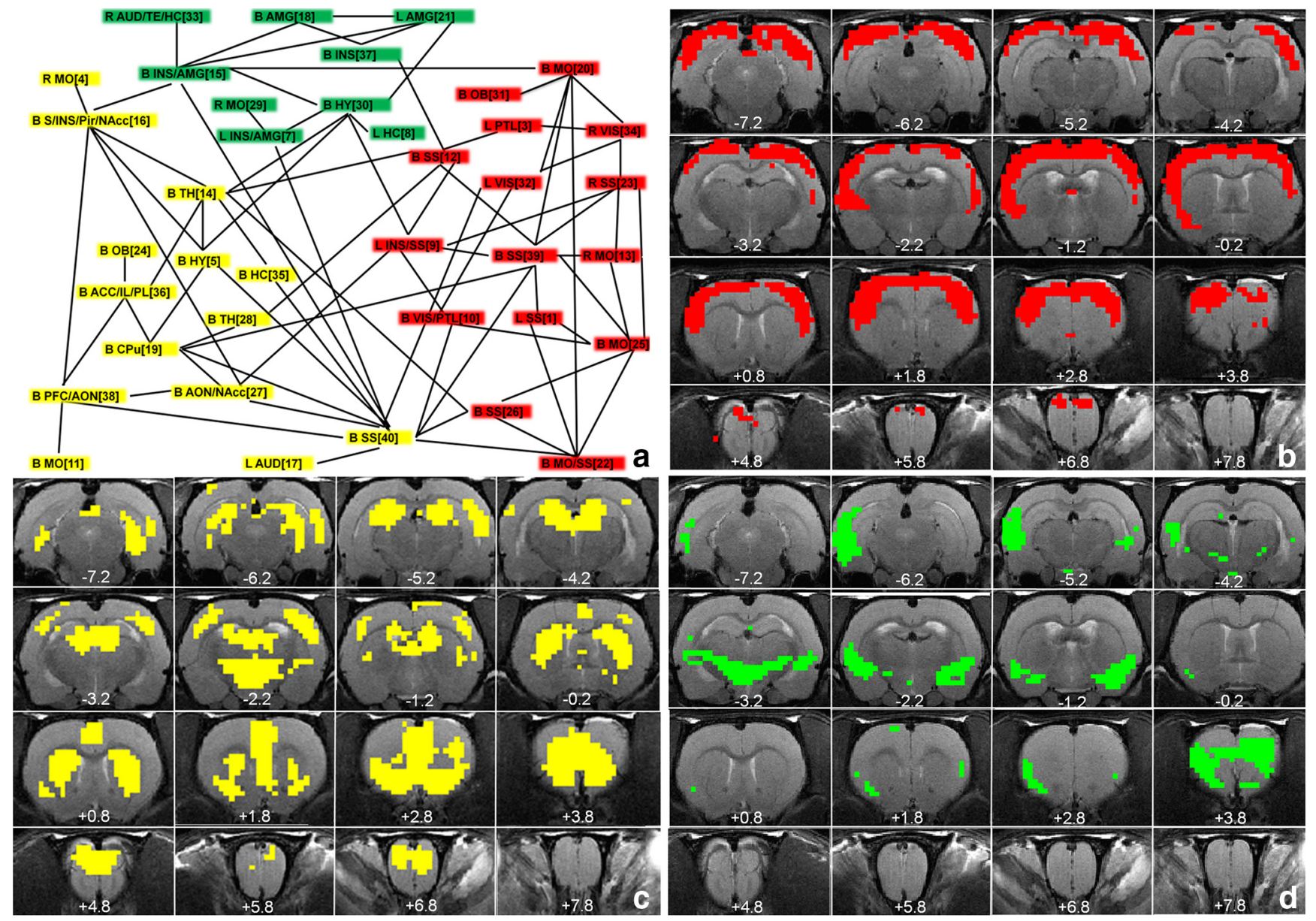

Figure 4. Segregation of the whole-brain network of the awake rat brain. $\boldsymbol{a}$, The global functional network constructed based on significant intercomponent connections. Each colored box represents an ICA component labeled with its corresponding anatomy and the ICA number. Each line represents a significant connection between two components. Nodes within the same module are displayed in the same color (red, green, and yellow). Three modules were obtained by the spectral partitioning algorithm. B, Bilateral; L, left; R, right; AMG, amygdala; INS, insula; NAcc, nucleus accumbens; M0, motor cortex; $\mathrm{HC}$, hippocampus; $\mathrm{HY}$, hypothalamus; $\mathrm{OB}$, olfactory bulb; Pir, piriform cortex; $\mathrm{PTL}$, parietal cortex; $\mathrm{S}$, septum; TE, temporal cortex; $\mathrm{TH}$, thalamus; VIS, visual cortex. $\boldsymbol{b}$ - $\boldsymbol{d}$, Community structures of the whole-brain network revealed by spectral partitioning. $\boldsymbol{b}$. The first module is dominated by cortical ribbon. $\boldsymbol{c}$, The second module is highlighted by the olfactory pathway and its interaction with PFC, and the integration of other sensory input, cognitive processing, and output in cortical and subcortical regions like thalamus and hippocampus. $\boldsymbol{d}$, The third module includes regions important for emotional and autonomic functions such as amygdala, insular cortex, PFC, and hypothalamus. The same colors are used in $\boldsymbol{b}, \boldsymbol{c}$, and $\boldsymbol{d}$ as those in $\boldsymbol{a}$. Distance to bregma (in millimeters) is labeled at the bottom of each image.

same distribution of degrees, showed similar results, $C / C_{\text {random }}=$ 1.5 and $L / L_{\text {random }}=1.02$. These comparisons collectively suggest that the rat brain is a small-world network (Watts and Strogatz, 1998).

\section{Discussion}

In this study, RSFC in awake rats was decomposed into 40 spatial components using group ICA. The direct connectional relationships between these components were evaluated using partial correlation, revealing a complex network linking different regions across the whole brain. This brain network was characterized by the features of small worldness with a large modularity, a large clustering coefficient, and a small shortest path length. Furthermore, using a graph-theory approach, the whole-brain network was segregated into community structures.

To our knowledge, this is the first study using group ICA to study RSFC in awake rats. ICA is well established in rsfMRI for decomposing functional clusters in the human brain. However, its application in the rat is rather limited. There is currently only one study that used ICA to analyze RSFC of individual, anesthetized rat without group analysis (Hutchison et al., 2010). Lack of such effort has significantly limited the applicability of rsfMRI, particularly in animal models. In the present study, images of all individual rats were aligned to a standard rat atlas, and thus allowed the group results to be obtained using group ICA. In addition, the awake condition avoided confounding effects of anesthesia. We found that the majority of components identified were located in anatomically well defined regions, indicating a convergence between anatomical parcellation and functional systems. Some components, such as bilateral somatosensory, motor, visual, and auditory cortices, are consistent with the literature (Peltier et al., 2005; Lu et al., 2007; Liu et al., 2010). Spatial maps of subcortical regions, including $\mathrm{CPu}$, thalamus, hypothalamus, and hippocampu,s also well agree with ICA results in individual anesthetized rats (Hutchison et al., 2010), suggesting highly reproducible patterns of cortical and subcortical clustering across individuals. However, we also observed several less reported yet important clusters. For instance, there were components related to olfactory and executive functions. Olfaction is considered one of the most important sensory inputs in the rodent. Prominent components of olfactory bulb, AON and OT indicated functional significance of olfaction in awake rats. Moreover, PFC and AON were clustered into a single component, suggesting a close asso- 

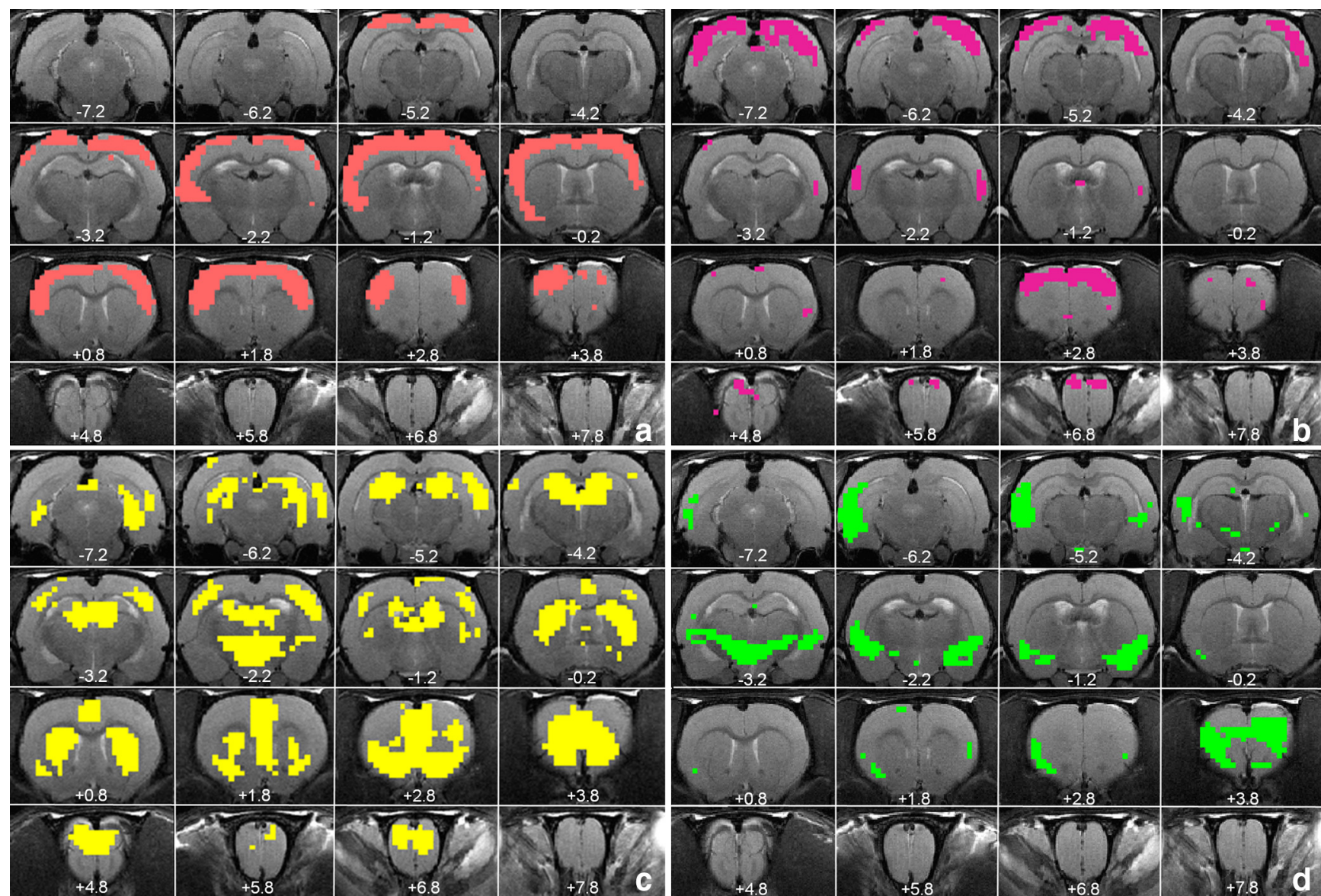

Figure 5. Community structures dominant in 20 repetitions of the spectral partitioning method combined with the fine-tuning method. Distance to bregma (in millimeters) is labeled at the bottom of each image. The yellow $(\boldsymbol{c})$ and green $(\boldsymbol{d})$ modules are almost identical to those shown in Figure 4 , whereas the module of cortical regions is further divided into two submodules $(\boldsymbol{a}, \boldsymbol{b})$.

ciation between olfactory and executive functions (Cinelli et al., 1987; Smith et al., 2010).

To further evaluate intercomponent connectional relationships, we applied partial correlation analysis on time courses of individual ICA components. Partial correlation analysis is an approach for estimating direct statistical association by controlling correlation mediated by other components. This analysis method essentially eliminated a large portion of connections that were mediated by other nodes, leaving only direct connections. A recent study evaluating various network modeling methods indicated that partial correlation performed very well in revealing network connections (Smith et al., 2010). In addition, this analysis could reveal possible long-distance functional integration. Significant amounts of direct connection identified in the present study are consistent with anatomical connections in the rat. For instance, the direct connection between thalamus and hippocampus observed in the present study has been well documented in the literature using various techniques (Wouterlood et al., 1990; Dolleman-Van Der Weel and Witter, 1996). These two regions and their bidirectional connections are critical components of the anatomical system subserving spatial memory (Henry et al., 2004). In addition, connections from the PFC to cingulate cortex and nucleus accumbens, as shown in our data, have been implicated in emotional processing (Hajós et al., 1998). We also observed that thalamus bridges hippocampus and ACC. In accordance with this result, it was found that nucleus reuniens of the midline thalamus might serve as the link sending projection to the hippocampus from the medial PFC, such as ACC (Vertes et al., 2007).
With the global functional network constructed based on intercomponent connections (Fig. 4a), the first question to consider is whether the rat brain exhibits the same network characteristics reported in humans, such as small-worldness. Human studies have indicated robust small-world characteristics in both structural and functional connectivity networks. A smallworld network is described as a high clustering coefficient and low minimum path length compared with random networks. Small-world networks allow high efficiency of information flow at a low wiring cost for both local (with a high clustering coefficient) and long distance (with a low minimum path length). Although small-worldness represents a crucial feature of brain organization in the human, there is a paucity of information regarding small-world networks in nonhuman subjects. Previous studies reported similar small-worldness of anatomical networks in the macaque visual cortex and cat whole cortex (Hilgetag et al., 2000). However, no study specifically addressed this question using functional connectivity in conscious rats. Our network metrics showed that, in the rat brain, the whole-brain network is considerably more cliquish than random networks, while retaining approximately the same minimum path length. These results are quantitatively comparable to the human brain and suggest that small-worldness is conserved in the rat functional networks.

In addition to the small-world features, high modularity is also thought to be an important governing principle in brain networks. Several studies consistently report that the restingstate brain network in humans exhibited robust community structure (He et al., 2009; Meunier et al., 2009). High modularity values of the rat whole-brain network obtained in our study in- 
dicate a robust community structure of the global network in the awake rat brain at the resting state. This result indicated that the rat brain shares basic topological characteristics with the human brain.

Using Newman's (2006) spectral partitioning method, the rat whole-brain network was segregated into three modules. The first module predominantly extended across the cortical ribbon, indicating a strong intercortical communication across the cortex (Zhang et al., 2010). The second module highlighted the olfactory pathway and its interaction with PFC, and the integration of other sensory input, cognitive processing, and output in cortical and subcortical regions. Regions in the third module, including PFC, insular cortex, hypothalamus, and amygdala, are all key components subserving emotional and autonomic regulations (Paxinos, 2004). Interestingly, using pharmacological MRI, Schwarz and colleagues (2009) reported very similar results with a module dominated by cortical regions and a second module dominated primarily with subcortical regions. Consistent with the intrinsic modular structure observed in the resting-state human brain, our rat results also showed long-distance interaction within modules.

To address the issue of degeneracy of the modularity function, distributions of $Q$ values and community structures were obtained. The result showed that two of the three modules previously identified (yellow and green) were highly consistent across all repetitions with little variation, whereas the community structure of cortical regions was further divided into two submodules. We speculate that the relatively lower stability of this module might reflect higher complexity of the organization of cortical networks.

The vertices in our graph are ICA components, as opposed to individual voxels or anatomically defined ROIs used in most other studies. The strategy of using ICA components to construct global networks is based on functionally segregated elements of the brain. Thus, we avoided anatomical restraint of ROI definitions. Recent evidence suggests that different anatomical parcellation schemes have significant influences on network topological properties (Wang et al., 2009) and functionally inaccurate ROIs could severely damage the network estimation (Smith et al., 2010). Therefore, our approach might have significant advantages in constructing the whole-brain network compared with anatomical ROI-based approaches. Relative to voxel-by-voxel approaches, our approach is more computationally efficient.

There are several methodological limitations of the present study. First, an unweighted network was used in graph-theory analysis. Future exploration on weighted networks should be interesting. Second, although rats were fully awake during RSFC scans, they were briefly anesthetized during setup. The effects of brief anesthesia on later RSFC need further investigation. Third, the ICA components number was arbitrary and other numbers can be used. In addition, negative intercomponent partial correlation coefficients (approximately half of all correlation coefficients) were not analyzed but can potentially contain important information regarding neural networks. This information should be taken into consideration in future studies. Furthermore, although intercomponent connectivity showed high consistency in the present study, individual variability particularly in topographical properties needs future examination.

Our understanding of the brain function has substantially benefited from preclinical neurobiological investigation in animal models, primarily in rodents. The present study systematically investigated resting-state functional networks in the awake rat brain. It provided a functional atlas of the intrinsic connec- tional architecture of the rat brain at both intraregion and interregion levels. More investigations are still needed to further characterize connectional architecture in the rat brain. For example, it is unknown whether functional networks in rats are organized differently at different spatial scales, or whether significant community structure exists within each module. It is also unknown whether the rat brain has the default mode network found in humans and primates (Raichle et al., 2001; Vincent et al., 2007). Nevertheless, the current work revealed that the conscious rat brain conserved topological properties like small-worldness also observed in human. Combined with various invasive procedures, pharmacological interventions, and genetic manipulations, it will serve as a prelude to future applications of RSFC in animal models.

\section{References}

Albert NB, Robertson EM, Miall RC (2009) The resting human brain and motor learning. Curr Biol 19:1023-1027.

Bassett DS, Bullmore ET (2009) Human brain networks in health and disease. Curr Opin Neurol 22:340-347.

Biswal B, Yetkin FZ, Haughton VM, Hyde JS (1995) Functional connectivity in the motor cortex of resting human brain using echo-planar MRI. Magn Reson Med 34:537-541.

Bullmore E, Sporns O (2009) Complex brain networks: graph theoretical analysis of structural and functional systems. Nat Rev Neurosci 10:186-198.

Calhoun VD, Adali T, Pearlson GD, Pekar JJ (2001) A method for making group inferences from functional MRI data using independent component analysis. Hum Brain Mapp 14:140-151.

Cinelli AR, Ferreyra-Moyano H, Barragan E (1987) Reciprocal functional connections of the olfactory bulbs and other olfactory related areas with the prefrontal cortex. Brain Res Bull 19:651-661.

Dolleman-Van Der Weel MJ, Witter MP (1996) Projections from the nucleus reuniens thalami to the entorhinal cortex, hippocampal field CA1, and the subiculum in the rat arise from different populations of neurons. J Comp Neurol 364:637-650.

Ferris CF, Febo M, Luo F, Schmidt K, Brevard M, Harder JA, Kulkarni P, Messenger T, King JA (2006) Functional magnetic resonance imaging in conscious animals: a new tool in behavioural neuroscience research. J Neuroendocrinol 18:307-318.

Fox MD, Raichle ME (2007) Spontaneous fluctuations in brain activity observed with functional magnetic resonance imaging. Nat Rev Neurosci 8:700-711.

Fox MD, Snyder AZ, Vincent JL, Corbetta M, Van Essen DC, Raichle ME (2005) The human brain is intrinsically organized into dynamic, anticorrelated functional networks. Proc Natl Acad Sci U S A 102:9673-9678.

Good BH, de Montjoye YA, Clauset A (2010) Performance of modularity maximization in practical contexts. Phys Rev E Stat Nonlin Soft Matter Phys 81:046106.

Greicius MD, Krasnow B, Reiss AL, Menon V (2003) Functional connectivity in the resting brain: a network analysis of the default mode hypothesis. Proc Natl Acad Sci U S A 100:253-258.

Greicius MD, Flores BH, Menon V, Glover GH, Solvason HB, Kenna H, Reiss AL, Schatzberg AF (2007) Resting-state functional connectivity in major depression: abnormally increased contributions from subgenual cingulate cortex and thalamus. Biol Psychiatry 62:429-437.

Hajós M, Richards CD, Székely AD, Sharp T (1998) An electrophysiological and neuroanatomical study of the medial prefrontal cortical projection to the midbrain raphe nuclei in the rat. Neuroscience 87:95-108

He Y, Wang J, Wang L, Chen ZJ, Yan C, Yang H, Tang H, Zhu C, Gong Q, Zang Y, Evans AC (2009) Uncovering intrinsic modular organization of spontaneous brain activity in humans. PLoS One 4:e5226.

Henry J, Petrides M, St-Laurent M, Sziklas V (2004) Spatial conditional associative learning: effects of thalamo-hippocampal disconnection in rats. Neuroreport 15:2427-2431.

Hilgetag CC, Burns GA, O’Neill MA, Scannell JW, Young MP (2000) Anatomical connectivity defines the organization of clusters of cortical areas in the macaque monkey and the cat. Philos Trans R Soc Lond B Biol Sci 355:91-110.

Horovitz SG, Braun AR, Carr WS, Picchioni D, Balkin TJ, Fukunaga M, Duyn 
JH (2009) Decoupling of the brain's default mode network during deep sleep. Proc Natl Acad Sci U S A 106:11376-11381.

Hutchison RM, Mirsattari SM, Jones CK, Gati JS, Leung LS (2010) Functional networks in the anesthetized rat brain revealed by independent component analysis of resting-state FMRI. J Neurophysiol 103: $3398-3406$.

Kennedy DP, Redcay E, Courchesne E (2006) Failing to deactivate: resting functional abnormalities in autism. Proc Natl Acad Sci USA 103:8275-8280

King JA, Garelick TS, Brevard ME, Chen W, Messenger TL, Duong TQ, Ferris CF (2005) Procedure for minimizing stress for fMRI studies in conscious rats. J Neurosci Methods 148:154-160.

Liu X, Zhu XH, Zhang Y, Chen W (2010) Neural origin of spontaneous hemodynamic fluctuations in rats under burst-suppression anesthesia condition. Cereb Cortex 21:374-384.

Liu Y, Liang M, Zhou Y, He Y, Hao Y, Song M, Yu C, Liu H, Liu Z, Jiang T (2008) Disrupted small-world networks in schizophrenia. Brain 131:945-961.

Lu H, Zuo Y, Gu H, Waltz JA, Zhan W, Scholl CA, Rea W, Yang Y, Stein EA (2007) Synchronized delta oscillations correlate with the resting-state functional MRI signal. Proc Natl Acad Sci U S A 104:18265-18269.

Massimini M, Ferrarelli F, Huber R, Esser SK, Singh H, Tononi G (2005) Breakdown of cortical effective connectivity during sleep. Science 309:2228-2232.

Meunier D, Lambiotte R, Fornito A, Ersche KD, Bullmore ET (2009) Hierarchical modularity in human brain functional networks. Front Neuroinformatics 3:37.

Newman ME (2006) Modularity and community structure in networks. Proc Natl Acad Sci U S A 103:8577-8582.

Pawela CP, Biswal BB, Cho YR, Kao DS, Li R, Jones SR, Schulte ML, Matloub HS, Hudetz AG, Hyde JS (2008) Resting-state functional connectivity of the rat brain. Magn Reson Med 59:1021-1029.

Paxinos G (2004) The rat nervous system. Sydney: Elsevier Academic.

Peltier SJ, Kerssens C, Hamann SB, Sebel PS, Byas-Smith M, Hu X (2005)
Functional connectivity changes with concentration of sevoflurane anesthesia. Neuroreport 16:285-288.

Raichle ME, MacLeod AM, Snyder AZ, Powers WJ, Gusnard DA, Shulman GL (2001) A default mode of brain function. Proc Natl Acad Sci U S A 98:676-682.

Rubinov M, Sporns O (2010) Complex network measures of brain connectivity: uses and interpretations. Neuroimage 52:1059-1069.

Schwarz AJ, Gozzi A, Bifone A (2009) Community structure in networks of functional connectivity: resolving functional organization in the rat brain with pharmacological MRI. Neuroimage 47:302-311.

Smith SM, Miller KL, Salimi-Khorshidi G, Webster M, Beckmann CF, Nichols TE, Ramsey JD, Woolrich MW (2010) Network modelling methods for FMRI. Neuroimage 54:875-891.

Stevens WD, Hasher L, Chiew KS, Grady CL (2008) A neural mechanism underlying memory failure in older adults. J Neurosci 28:12820-12824.

Vertes RP, Hoover WB, Szigeti-Buck K, Leranth C (2007) Nucleus reuniens of the midline thalamus: link between the medial prefrontal cortex and the hippocampus. Brain Res Bull 71:601-609.

Vincent JL, Patel GH, Fox MD, Snyder AZ, Baker JT, Van Essen DC, Zempel JM, Snyder LH, Corbetta M, Raichle ME (2007) Intrinsic functional architecture in the anaesthetized monkey brain. Nature 447:83-86.

Wang J, Wang L, Zang Y, Yang H, Tang H, Gong Q, Chen Z, Zhu C, He Y (2009) Parcellation-dependent small-world brain functional networks: a resting-state fMRI study. Hum Brain Mapp 30:1511-1523.

Wang J, Zuo X, He Y (2010) Graph-based network analysis of resting-state functional MRI. Front Syst Neurosci 4:16.

Watts DJ, Strogatz SH (1998) Collective dynamics of 'small-world' networks. Nature 393:440-442.

Wouterlood FG, Saldana E, Witter MP (1990) Projection from the nucleus reuniens thalami to the hippocampal region: light and electron microscopic tracing study in the rat with the anterograde tracer Phaseolus vulgaris-leucoagglutinin. J Comp Neurol 296:179-203.

Zhang N, Rane P, Huang W, Liang Z, Kennedy D, Frazier JA, King J (2010) Mapping resting-state brain networks in conscious animals. J Neurosci Methods 189:186-196. 